\title{
The Geometry of Power Systems Steady-State Equations- Part I: Power Surface
}

\author{
B.I. Ayuev ${ }^{1}$, V.V Davydov ${ }^{2,3,}$, , P.M. Erokhin ${ }^{1,4}$, V.G. Neuymin ${ }^{5}$, A.V. Pazderin ${ }^{4}$
}

${ }^{1}$ JSC "System Operator of the Unified Power System," Moscow, Russia.

${ }^{2}$ Branch of JSC "System Operator of the Unified Power System" United dispatching department of the power system of Siberia, Kemerovo, Russia.

${ }^{3}$ East Siberia State University of Technology and Management, Ulan-Ude, Russia.

${ }^{4}$ Ural Federal University, Yekaterinburg, Russia.

${ }^{5}$ JSC "Scientific and Technical Center of Unified Power System", Saint Petersburg, Russia.

\begin{abstract}
Steady-state equations play a fundamental role in the theory of power systems and computation practice. These equations are directly or mediately used almost in all areas of the power system state theory, constituting its basis. This two-part study deals with a geometrical interpretation of steady-state solutions in a power space. Part I considers steady states of the power system as a surface in the power space. A power flow feasibility region is shown to be widely used in power system theories. This region is a projection of this surface along the axis of a slack bus active power onto a subspace of other buses power. The findings have revealed that the obtained power flow feasibility regions, as well as marginal states of the power system, depend on a slack bus location. Part II is devoted to an analytical study of the power surface of power system steady states.
\end{abstract}

Index Terms: feasibility region, Jacobian, marginal state, power flow, power surface, power system, slack bus, steady state.

\section{INTRODUCTION}

STEADY-state calculations play a crucial part in the analysis, planning, and control of power systems. The majority of research papers published thus far focus on the development of numerical methods, which are fairly completely reviewed in [1] and [2]. At the same time, an analytical investigation and understanding of the

\footnotetext{
* Corresponding author.

E-mail: davv@bur.so-ups.ru
}

http://dx.doi.org/10.38028/esr.2020.03.0005

Received May 17, 2020. Revised June 23, 2020.

Accepted July 13, 2020. Available online December 27, 2020.

This is an open access article under a Creative Commons Attribution-NonCommercial 4.0 International License.

(C) 2020 ESI SB RAS and authors. All rights reserved. steady-state equations are essential since they provide qualitative insights [3].

Initially, a steady-state solution space was examined for lossless power systems with $P V$-buses only. The authors of [4] indicate that the steady-state solution space is bounded by the power flow feasibility region in the parametric space; and that a steady-state solution may be non-unique, i.e., there can be several solutions, in some of which power circulates in one or more loops of the network. Using Hamiltonian formulation, the authors of [5] show that stable power flows are not necessarily unique either. A study of topological properties of the stable region [6] reveals that in such cases, the stable region is disconnected. The author of [7] uses Hamiltonian formulation to investigate the number of steady-state solutions, topological properties of stationary points, and features of a stable region for a 3-bus radial system.

Explorations of steady-state solution space for lossy power systems were usually confined to computational studies. The author of [8] has studied a limit on the existence of a steady-state solution of the 3-bus radial power system with $P V$-buses only. The influence the parameters of the system lines have on the power flow feasibility region was investigated in [9]. A computational study of the power flow feasibility region for a 3-bus radial power system with $P V$-buses only is presented in [10]. The author of [11] proposes using $L$-functions to study the power flow feasibility region, analytically shows, and computationally confirms that the power flow feasibility region can have a hole through it. An analytical framework is developed in [12] to describe the convexity properties of the power flow feasibility boundary in parametric space. In [13], the researchers propose a contour approach to the global analysis of power system performance, and in [14], a monogram method is developed to explore a solution boundary of the power flow problem.

It is noteworthy that the cited scientific studies, as well as others, tend to use geometrical images only to represent 
numerical results [15] or to explain the proposed approaches [17], [18], [16]. At the same time, the development of the bifurcation theory [19], singularity theory [20], and catastrophe theory [21], [22] shows that the geometric consideration provides qualitatively new results.

The objective of the present paper is to propose a geometric interpretation of a set of all the solutions to steady-state equations in a power space. The rest of the paper is organized as follows. Section II presents an analysis of specific properties of steady-state equations. Section III proposes and considers the geometric interpretation of the steady-state solutions, i.e., a power surface, and reveals its relation to the feasibility regions of power flow and marginal states of power systems. Section IV discusses the causes of the hole inside a power flow feasibility region. Section V geometrically justifies the slack bus application in steady-state calculations. Section VI presents the conclusions.

\section{Specific Properties of A Steady-StATE MODEL}

There are several steady-state models, each of which is based on the Kirchhoff laws, has certain advantages and shortcomings, and can be obtained from another one by a mathematical transformation. Therefore, qualitative results and conclusions obtained for the model of interest remain valid for all other steady-state models. Consider the nodal steady-state equations in polar coordinates:

$$
\begin{gathered}
\Delta P_{k}=P_{k}+\sum_{m} V_{k} V_{m}\left|Y_{k m}\right| \sin \left(\delta_{k m}-\alpha_{k m}\right)=0 ; \\
\Delta Q_{k}=Q_{k}-\sum_{m} V_{k} V_{m}\left|Y_{k m}\right| \cos \left(\delta_{k m}-\alpha_{k m}\right)=0,
\end{gathered}
$$

where $P_{k}, Q_{k}, \delta_{k}, V_{k}$ are active and reactive power, the voltage angle and magnitude at bus $k$, respectively; $Y_{k m}=G_{k m}+j B_{k m}$ is an element of the bus admittance matrix; $\alpha_{k m}=-\operatorname{arctg}\left(G_{k m} / B_{k m}\right)$ is a loss angle; $\delta_{k m}=\delta_{k}-\delta_{m}$.

The steady-state calculation involves solving the set of nonlinear simultaneous equations (1), under the assumption that the system parameters, i.e., bus admittance matrix elements, are known, and a steady state at each bus is determined by four parameters (variables) $-P_{k}, Q_{k}$, $\delta_{k}$, and $V_{k}$. According to (1), each bus has two balance equations for active and reactive power. Therefore, to obtain a solution, two of four variables $P_{k}, Q_{k}, \delta_{k}, V_{k}$, are to be specified at each bus. Therefore, the system of nonlinear equations (1) can be summed up as follows:

$$
\Delta F(X, Y)=0,
$$

where $\Delta F(X, Y)$, and $X, Y$, are the vector of power mismatches and the vectors of dependent and independent (given) variables, respectively. Mathematically, there is no special way to determine the dependent and independent variables. Only the number of the dependent variables is given. In terms of the steady-state solution, the independent variables are those that can be controlled [2], for example, power injections and voltage magnitudes at the generation buses with automatic excitation control. Buses voltage are dependent variables obtained by solving (2).
Solving the system of nonlinear equations (2) can be considered as mapping the independent variables into a space of dependent variables $X=X(Y)$ [23]. According to the implicit function theorem [20], the necessary condition for the existence of such a map (as well as the solution existence) is the non-singularity of a matrix of first partial derivatives of the nonlinear equations set with respect to dependent variables $[\partial \Delta F / \partial X]$.

Consider a full matrix of first partial derivatives (Jacobian) of the nodal steady states equations with respect to the voltage angles and magnitudes:

$$
\left[J_{\mathrm{F}}\right]=\left|\begin{array}{ll}
\partial \Delta P / \partial \delta & \partial \Delta P / \partial V \\
\partial \Delta Q / \partial \delta & \partial \Delta Q / \partial V
\end{array}\right|
$$

where

$$
\begin{aligned}
& \left(\frac{\partial \Delta P}{\partial \delta}\right)_{k m}=\left\{\begin{array}{l}
-V_{k} V_{m}\left|Y_{k m}\right| \cos \left(\delta_{k m}-\alpha_{k m}\right), \quad k \neq m ; \\
\sum_{m \neq k} V_{k} V_{m}\left|Y_{k m}\right| \cos \left(\delta_{k m}-\alpha_{k m}\right), \quad k=m ;
\end{array}\right. \\
& \left(\frac{\partial \Delta P}{\partial V}\right)_{k m}=\left\{\begin{array}{l}
V_{k}\left|Y_{k m}\right| \sin \left(\delta_{k m}-\alpha_{k m}\right), \quad k \neq m ; \\
2 V_{k} \mathrm{G}_{k k}+\sum_{m \neq k} V_{m}\left|Y_{k m}\right| \sin \left(\delta_{k m}-\alpha_{k m}\right), k=m ;
\end{array}\right. \\
& \left(\frac{\partial \Delta Q}{\partial \delta}\right)_{k m}= \begin{cases}-V_{k} V_{m}\left|Y_{k m}\right| \sin \left(\delta_{k m}-\alpha_{k m}\right), \quad k \neq m ; \\
\sum_{m \neq k} V_{k} V_{m}\left|Y_{k m}\right| \sin \left(\delta_{k m}-\alpha_{k m}\right), \quad k=m ;\end{cases} \\
& \left(\frac{\partial \Delta Q}{\partial V}\right)_{k m}=\left\{\begin{array}{l}
-V_{k}\left|Y_{k m}\right| \cos \left(\delta_{k m}-\alpha_{k m}\right), \quad k \neq m ; \\
2 V_{k} B_{k k}-\sum_{m \neq k} V_{m}\left|Y_{k m}\right| \cos \left(\delta_{k m}-\alpha_{k m}\right), k=m .
\end{array}\right.
\end{aligned}
$$

Equations (3)-(4) show that the diagonal elements of $[\partial \Delta P / \partial \delta]$ and $[\partial \Delta Q / \partial \delta]$ are equal to the sum of the nondiagonal row elements with an opposite sign. Therefore $[\partial \Delta P / \partial \delta] e=0,[\partial \Delta Q / \partial \delta] e=0$, where $e$ is the vector of all ones. Hence the full Jacobian $\left[J_{F}\right]$ will be singular since $\left[J_{F}\right]\left[e^{\mathrm{T}}, 0^{\mathrm{T}}\right]^{\mathrm{T}}=\left[0^{\mathrm{T}}, 0^{\mathrm{T}}\right]^{\mathrm{T}}$.

This property of the full Jacobian (3) is a direct consequence of (1). Changes in every voltage angle by the same value does not influence its left-hand and righthand sides. Therefore, system (1) has an infinite number of solutions. To specify a solution, it is necessary to set a reference point, i.e., the voltage angle of a bus should be considered as known, i.e., this voltage angle must be moved from dependent variables to independent ones. Such a bus is called the angle reference bus. Since the number of dependent variables is reduced by one, mathematically, the initial number of dependent variables should be restored. Due to the power system-specific features, the active power of a bus is assigned as a new dependent variable. This bus balances active power in the whole power system and is called a slack bus. The reference bus may be chosen arbitrarily. The slack bus is chosen based on the specific features of a power system.

In general, two other sub-matrices $[\partial \Delta P / \partial V]$ and $[\partial \Delta Q / \partial V]$ of the full Jacobian are not singular. However, if there are no shunts, transformer tap ratios, and phase shifters, their diagonal elements at the point of "flat start" 
are also equal to the sum of non-diagonal row elements with opposite sign, i.e., $[\partial \Delta P / \partial V] e=0,[\partial \Delta Q / \partial V] e=0$. In this case, $\left[J_{F}\right]\left[0^{\mathrm{T}}, e^{\mathrm{T}}\right]^{\mathrm{T}}=\left[0^{\mathrm{T}}, 0^{\mathrm{T}}\right]^{\mathrm{T}}$ and a null-space dimension of the full Jacobian will be equal to two. The use of the reference and slack buses reduces the null-space dimension of the full Jacobian by one, but the Jacobian remains singular. In most cases, voltage magnitudes are not equal to the «flat start» values; therefore, submatrices $[\partial \Delta P / \partial V]$ and $[\partial \Delta Q / \partial V]$ are not singular. They are, however, ill-conditioned, and the resulting solution is very sensitive to reactive power variation. As a result, it is almost impossible to set the reactive power of buses so that the resulting steady states correspond to the power system operating condition [24]. Therefore, it is necessary to fix the voltage magnitude at one of the buses, i.e., to make it an independent variable. As in the case of fixing voltage angle, the reactive power at one of the buses is assigned as a new dependent variable. Therefore, this bus becomes the bus balancing reactive power. The slack bus balances both active and reactive power ( $V \delta$-bus). Generator buses equipped with automated excitation control balance reactive power as well. These buses keep voltage magnitudes constant by regulating reactive power within given ranges ( $P V$-buses).

With all the above things considered, the matrix of first partial derivatives of steady-state equations with respect to dependent variables can have the following form:

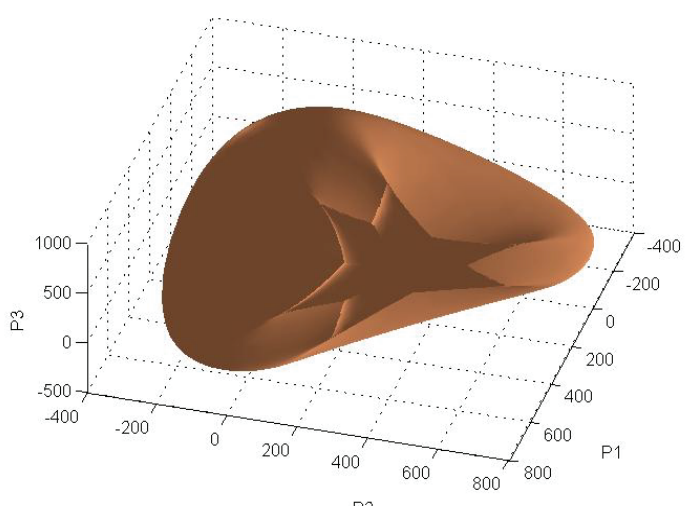

(a)

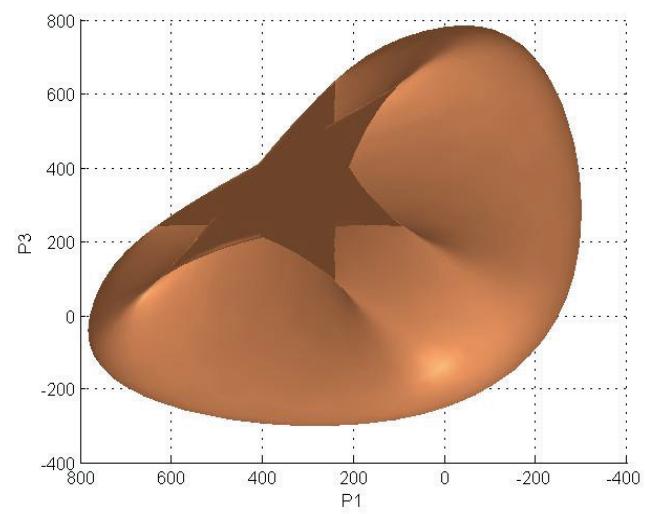

(c)

$$
[J]=\left[\begin{array}{cccc}
J_{P F} & 0 & 0 \\
\partial \Delta P_{b} / \partial \delta & \partial \Delta P_{b} / \partial V_{P Q} & 1 & 0 \\
\partial \Delta Q_{P V} / \partial \delta & \partial \Delta Q_{P V} / \partial V_{P Q} & 0 & \mathrm{E}
\end{array}\right],
$$

where

$$
\left[J_{\mathrm{PF}}\right]=\left[\begin{array}{cc}
\partial \Delta P / \partial \delta & \partial \Delta P / \partial V_{P Q} \\
\partial \Delta Q_{P Q} / \partial \delta & \partial \Delta Q_{P Q} / \partial V_{P Q}
\end{array}\right]
$$

is the standard power flow Jacobian; [E] and [0] are the identity and zero sub-matrices of respective sizes, and index $b$ is used for the slack bus. According to (5) $\operatorname{det}[J]=\operatorname{det}\left[J_{P F}\right]$ and non-singularity of power flow Jacobian, (6) ensures non-singularity of matrix (5).

Formally, to solve the system of nonlinear equations (1) by the Newton method, the system of linearized equations with matrix (5) is to be solved. However, the linearized equations using $\left[J_{P F}\right]$ do not depend on other equations and are solved separately. All the other dependent variables, e.g., the power at slack bus and reactive power at $P V$ buses, are determined by (1) with ordinary substitution of voltage angles and magnitudes.

The active and reactive power of the corresponding buses is included in (1) additionally. Excluding technical limitations, system (1) will always be consistent with any voltage angles and magnitudes, if the power at buses is obtained directly using (1). Therefore, a marginal state of

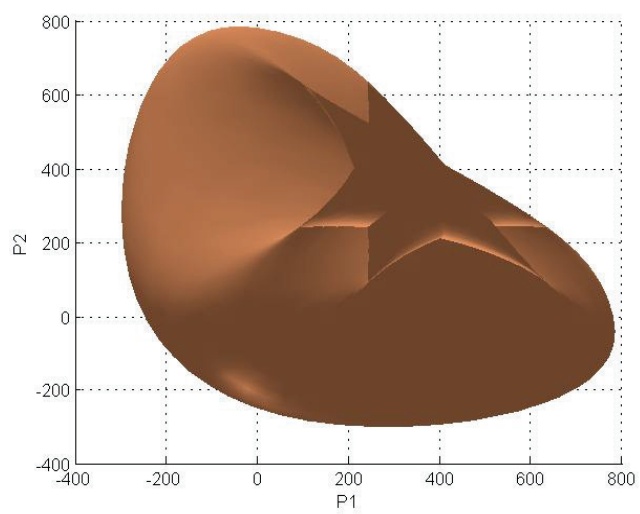

(b)

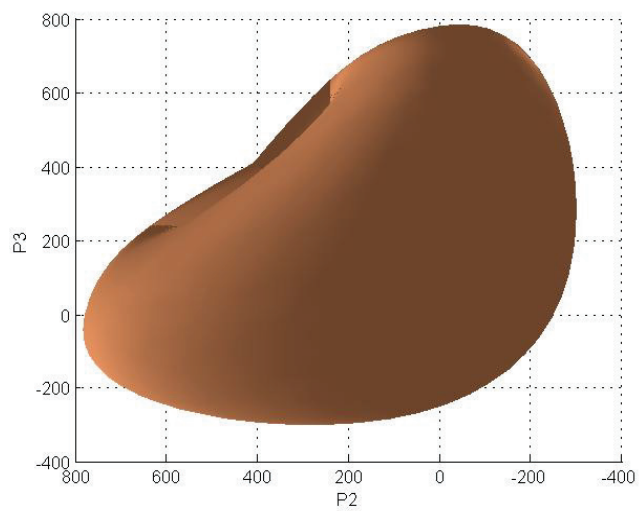

(d)

Fig. 1. Power surface of a 3-bus system (a) and its projections onto coordinate planes (b)-(d). 


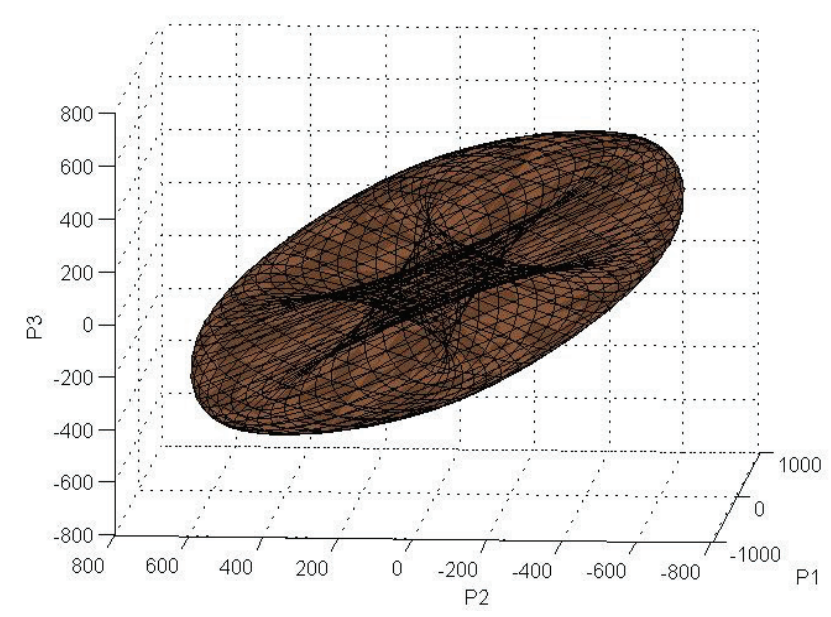

(a)

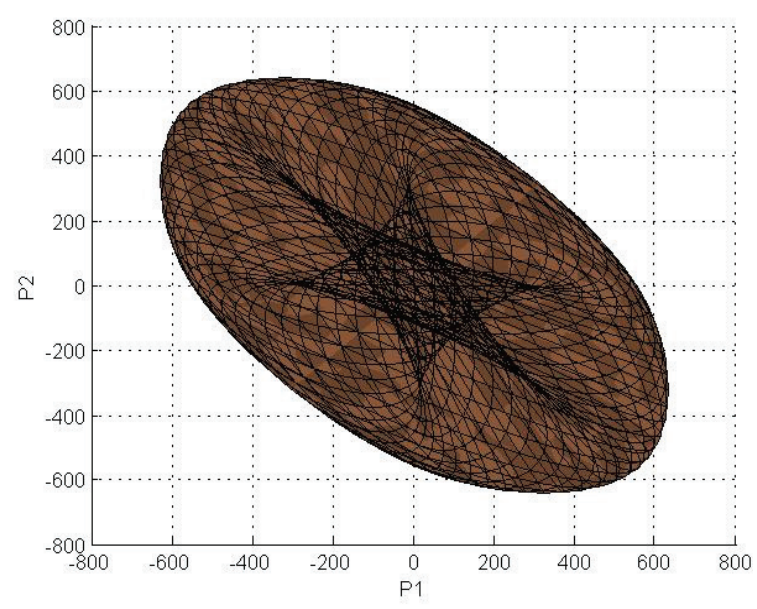

(b)

Fig. 2. Power surface (a) and power flow feasibility region (b) of 3-bus lossless system

a power system is a steady state where small deviations of independent variables in an unfavorable direction lead to inconsistency of system (1), i.e., it cannot be solved with respect to dependent variables. The implicit function theorem asserts that if Jacobian (5) at point $X_{0}, Y_{0}$ is not singular, then for each $Y$ close enough to $Y_{0}$ there is only one solution $X=X(Y)$, which in turn is the solution to the nonlinear nodal steady-state equations $\Delta F(X(Y), Y)=0$. Therefore, corollaries of the implicit function theorem are: firstly, a necessary condition (criterion) for the marginal states is the singularity of (5)-(6) [25]; secondly, another necessary condition is the existence of close alternative solutions nearby the marginal state [20]. According to (5)-(6), the Jacobian depends on the set of dependent and independent variables of the steady-state model. Therefore, slack bus location influences the power flow Jacobian and MS [26].

It is necessary to note an important property of the power flow Jacobian. According to the Sard theorem, a set of non-regular values of variables, i.e., when the Jacobian is singular, has measure zero [23]. In terms of the probability theory, it means that the probability of such events is equal to zero. The complement for every set of measure zero is dense everywhere, i.e., the set of regular values has a full measure. Therefore, each point in the space of variables is arbitrarily close to some regular value of the map. On the one hand, the Sard theorem guarantees the impossibility of the practical existence of a normal steady state having the singular Jacobian, i.e., makes the necessary condition of marginal state the sufficient one. On the other hand, it asserts that the obtained solutions are only approximations of actual marginal states. Mathematicians seldom apply the Sard theorem. However, it is a very powerful and useful mathematical tool for those who elaborate or apply computing models of power systems. For example, the theorem allows explaining the operability of the damped Newton method when the steady-state equations have no real solution. It is known that in this case, the iteration process of the damped Newton method converges to a marginal state with a «singular» Jacobian [27], [28]. Theoretically, it is impossible to solve a system of linear equations with a singular matrix. However, this is done by the damped Newton method [2]. It confirms that in computing models, the power flow Jacobian is not singular, although it may be ill-conditioned. In the damped Newton method, an optimal multiplier considerably improves the condition number of the problem to be solved.

\section{POWER SURFACE}

Consideration of solving the steady-state equations as mapping of independent variables onto the space of dependent variables $Y \rightarrow X$ allows obtaining an interesting and important geometrical interpretation of steadystate equations. Since the slack bus active power $P_{b}$ is a dependent variable, it is a function of the active power of all other buses and the reactive power of the $P Q$-buses. Its graph $P_{b}=P_{b}(P, Q)$ is a surface in the power space. The power surface defines the set of the power system steady states, including hypothetical unstable ones, for given system parameters [29]. There are no power system steady states outside the power surface for the specified system parameters and independent variables. Any point of the power surface corresponds to a steady state and its coordinates, i.e., the power at the buses in this steady state. As an example, Fig. 1 shows the power surface of a 3-bus power system with all $P V$-buses and its projections onto the corresponding coordinate planes. Parameters of this power system are as follows:

$$
V_{1}=V_{2}=V_{3}=110 \mathrm{kV} ; Z_{12}=Z_{13}=Z_{23}=20+j 40 \Omega \text {. }
$$

The power system state analysis tends to use the concept of the power flow feasibility region. As the power surface determines all the power system steady states for the given system parameters, its projection along the axis of the slack bus active power onto a subspace of power of 


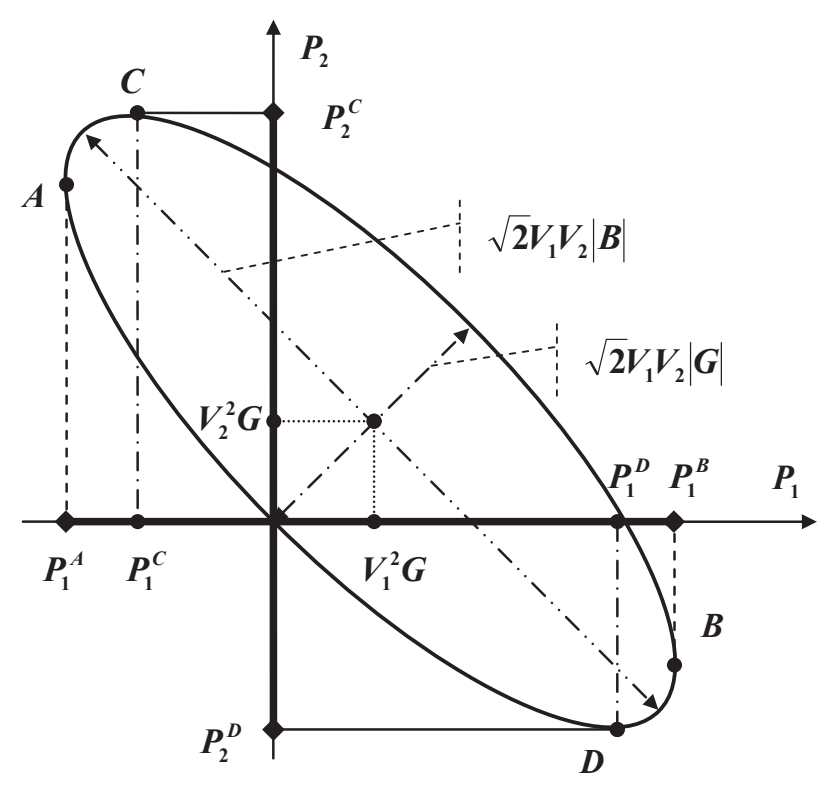

Fig. 3. Power ellipse of 2-bus power system

all other buses is nothing else but the power flow feasibility region and boundary of this projection represents a marginal state surface in the power subspace of the buses. Thus, projections of the power surface presented in Fig. 1 (b), (c), or (d) are power flow feasibility regions when buses 3, 2, or 1 are used as slack buses, respectively. Since the power surface is normally not plane, its projection, i.e., the power flow feasibility region, and its boundary, i.e., the marginal state surface, will depend on the choice of the axis along which it is projected, i.e., they depend on the slack bus choice.

The power surface of a lossless power system is plane since there are no active power losses and power at buses satisfies the equation $\sum P_{k}=0$. As an example, Fig. 2(a) shows the power surface of a 3-bus lossless power system $\left(Z_{12}=Z_{13}=Z_{23}=40 \Omega\right)$ with $P V$-buses, and Fig. 2(b) indicates its projection onto the coordinate plane. This surface is a plane ellipsoid. The boundary of the plane surface corresponds to the marginal state, and only its projection along the axis of the active power of any slack bus onto the subspace of power at other buses is the boundary of the power flow feasibility region of the lossless power system. Therefore, the marginal states of lossless power systems do not depend on the choice of a slack bus.

It is known that the set of nonlinear steady-state equations for specified independent variables may have several solutions, some of which are stable while others are not. If the transmission lines of the power system are not purely inductive, then the number of solutions corresponds to the number of crossings of the power surface by the vector of the given buses' power directed in parallel to the axis of the slack bus active power. When there is purely inductive reactance, then even one power surface point may correspond to several solutions.

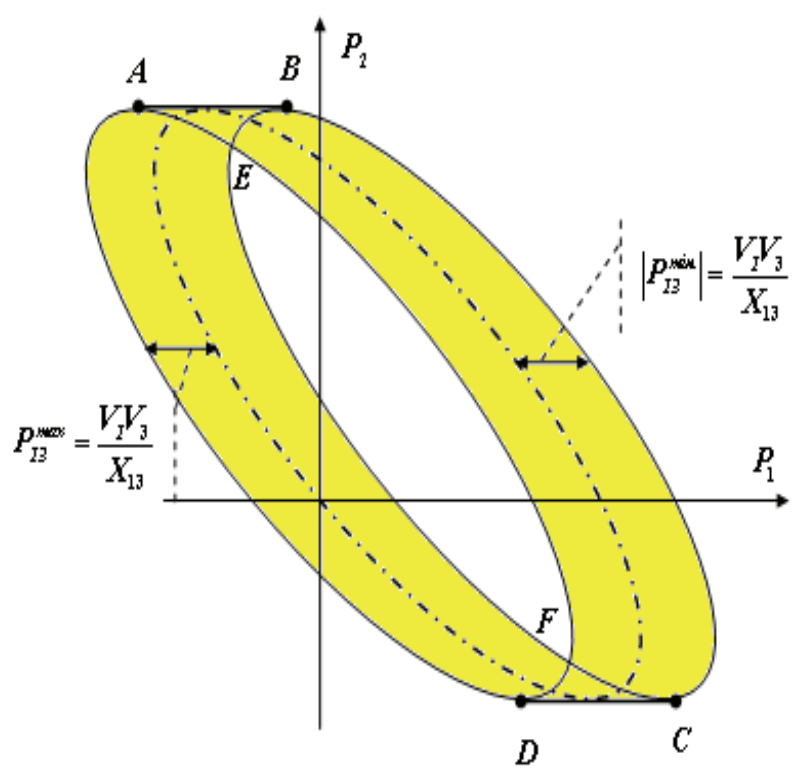

Fig. 4. Power flow feasibility region of 3-bus radial power system

Thus, each point of the plane power surface of the 3-bus lossless power system represented in Fig. 2 corresponds to one solution on the boundary of the power surface; two solutions inside the surface but outside the region of triangles; and two additional solutions inside each triangle region, but only one of these solutions is stable. In the area of crossing triangles, each power surface point corresponds to six solutions, including the origin [4], i.e., when power injections of all the buses are equal to zero. Four solutions, including a stable one, correspond to zero active power flows in the lines. The other two solutions correspond to the circulation of active power around the loop (even when all the active power injections are zero), giving the sum of the branch-angles along the loop equal to $\pm 360^{\circ}$, rather than zero as in an ordinary solution. According to [5] and [6], interconnected power systems may have stable solutions, where active power circulates in one or more loops. In these cases, losses are much larger than those for ordinary solution.

\section{Power Flow Feasibility Region with a Hole}

The geometrical consideration of the power system's steady states as the power surface allows highlighting the reasons why holes can occur inside the power flow feasibility region [11]. This can be done by considering the power surface of a 2-bus system with $P V$-buses presented in Fig. 3. This «surface» is a hollow ellipse in the two-dimensional space of bus power [32]

$$
\left(\frac{P_{1}-V_{1}^{2} G+P_{2}-V_{2}^{2} G}{2 V_{1} V_{2} \mathrm{G}}\right)^{2}+\left(\frac{P_{1}-V_{1}^{2} G-P_{2}+V_{2}^{2} G}{2 V_{1} V_{2} \mathrm{~B}}\right)^{2}=1,
$$

where $G+j B$ is the complex admittance of the line connecting these two buses. 


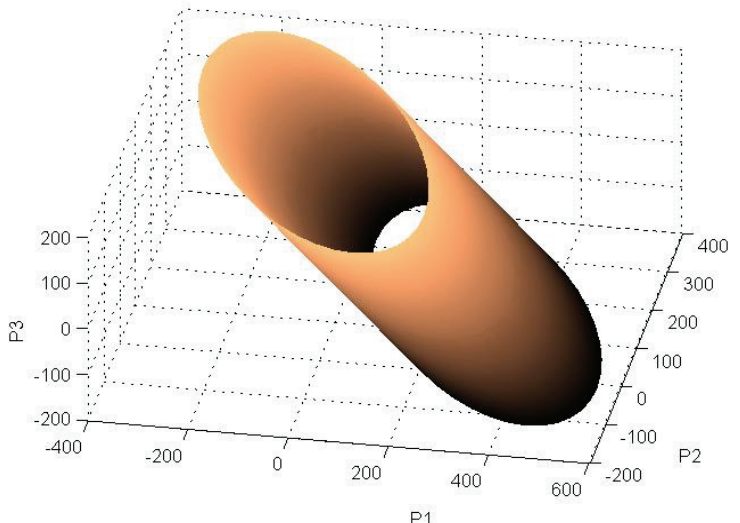

(a)

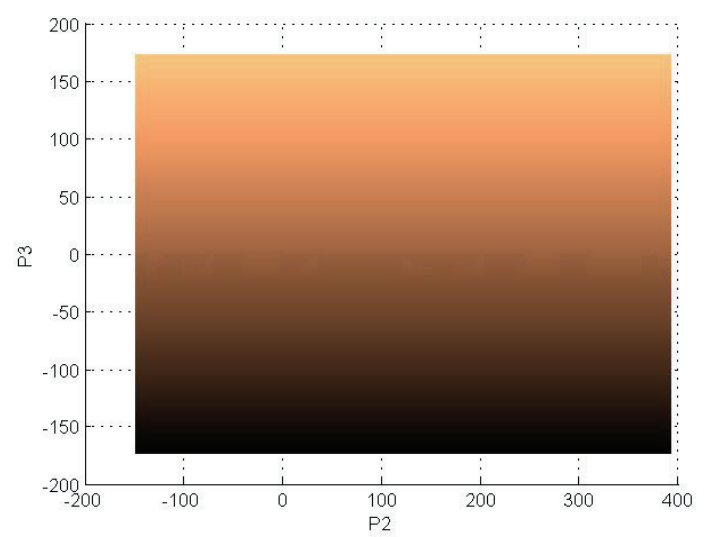

(c)

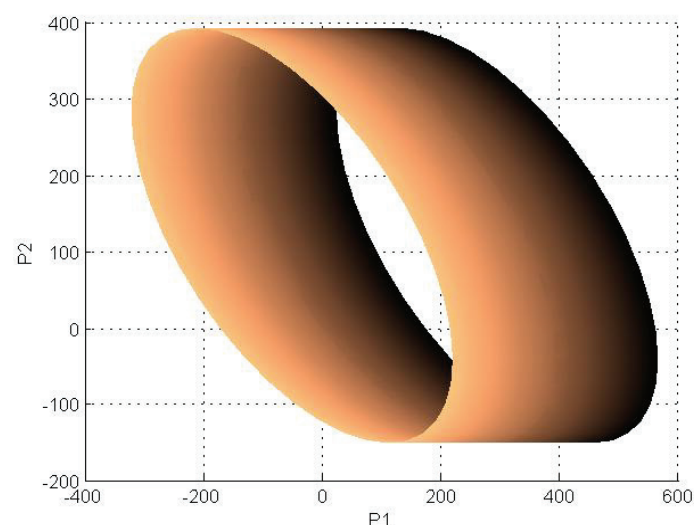

(b)

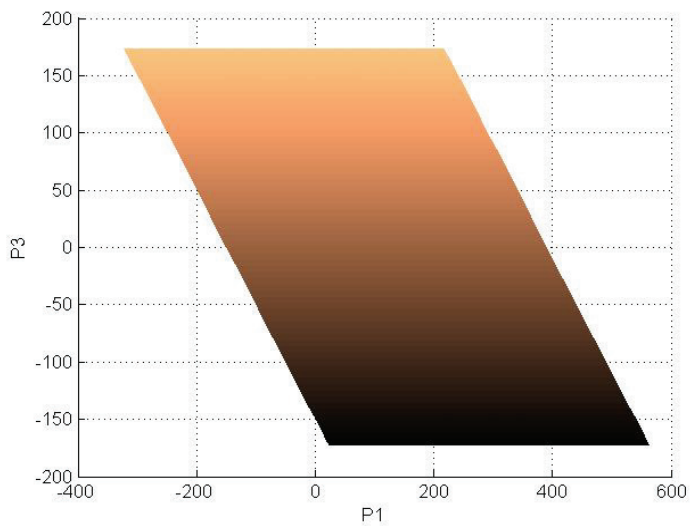

(d)

Fig. 5. Power surface of a 3-bus radial system (a) and power flow feasibility regions when the slack bus is bus 3 (b), bus 1 (c), or bus 2 (d).

The coordinates of the center of the power ellipse are $\left(V_{1}^{2} G, V_{2}^{2} G\right)$ and its principal axes are rotated by an angle of $45^{\circ}$ relative to the coordinate axes. The length of one of the principal axes is $\sqrt{2} V_{1} V_{2}|\mathrm{~B}|$, the length of the other is $\sqrt{2} V_{1} V_{2}|\mathrm{G}|$. The power ellipse defines all the steady states of the given 2-bus system. Any ellipse point corresponds to a particular steady state, and its coordinates are steady-state power at the buses. Outside and inside this hollow ellipse, there are no steady states. Therefore, a set of feasible power of the buses for the 2-bus system is non-convex. It is one of the principal prerequisites for the holes to appear inside power flow feasibility regions.

Note that power at all buses is equal in rights for the power surface, and a slack bus is not required. The need to use the slack bus arises in the case of steady-state calculation. If bus 2 is used as a slack bus to calculate the steady state for the 2-bus system, the thick line $\left[P_{1}^{A}, P_{1}^{B}\right]$ in Fig. 3, which is the projection of the power ellipse onto the active power axis of bus 1, will be the power flow feasibility region. In this case, points $A$ and $B$ correspond to two marginal states. On the other hand, if bus 1 is a slack bus, the thick line $\left[P_{2}^{C}, P_{2}^{D}\right]$ in Fig. 3, which is the projection of the power ellipse onto the active power axis of bus 2, will be another power flow feasibility region, where points $C$ and $D$ will correspond to two other marginal states.
The power flow feasibility region of the 2-bus power system is a straight-line segment and, therefore, has no holes. However, a hole in the power flow feasibility region may arise already in the case of a 3-bus system. To see it, add $P V$-bus 3 connecting the 2-bus system to bus 1 , for example, by the inductive impedance $Z_{13}=j X_{13}$, and assign bus 3 as a slack bus. In this radial 3-bus power system, all buses are $P V$-buses, and the active power of bus 3 is directly transported to bus 1 without power losses. Therefore, using (7), the power surface of this system can be determined by

$$
\begin{gathered}
\left(\frac{P_{1}+P_{3}-V_{1}^{2} G+P_{2}-V_{2}^{2} G}{2 V_{1} V_{2} \mathrm{G}}\right)^{2}+ \\
+\left(\frac{P_{1}+P_{3}-V_{1}^{2} G-P_{2}+V_{2}^{2} G}{2 V_{1} V_{2} \mathrm{~B}}\right)^{2}=1
\end{gathered}
$$

where $P_{3}=P_{31}=\left(V_{1} V_{3} / X_{13}\right) \sin \delta_{31}$.

Expression (8) is the equation of a surface in a threedimensional space, and its projection onto the coordinate plane $P_{1} \times P_{2}$ represents a power flow feasibility region for this power system when bus 3 is used as a slack bus (Fig. 4). Comparison of (8) with (7) reveals that the ellipse (8) for particular $P_{3}$ is the same ellipse (7) as in Fig. 3, but displaced horizontally to the left by the value of power $P_{3}$ in the case where bus 1 receives power from slack bus 3 , 


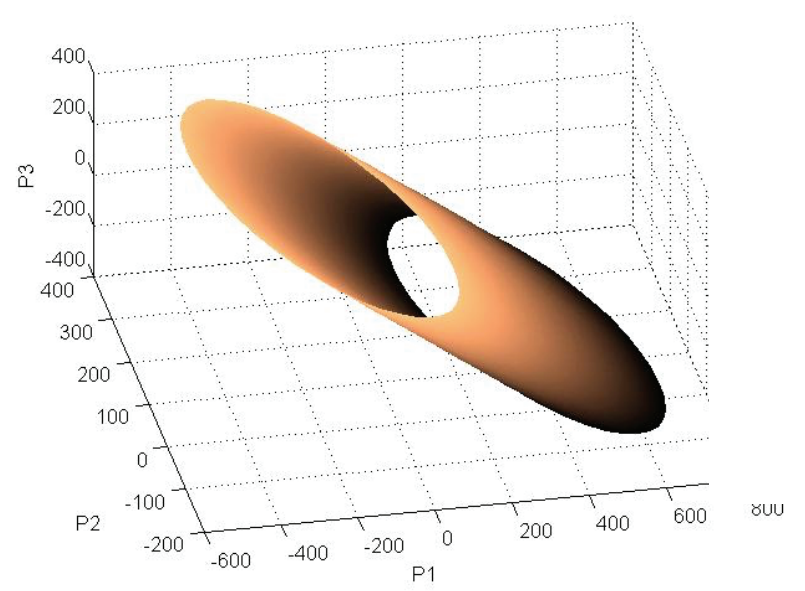

(a)

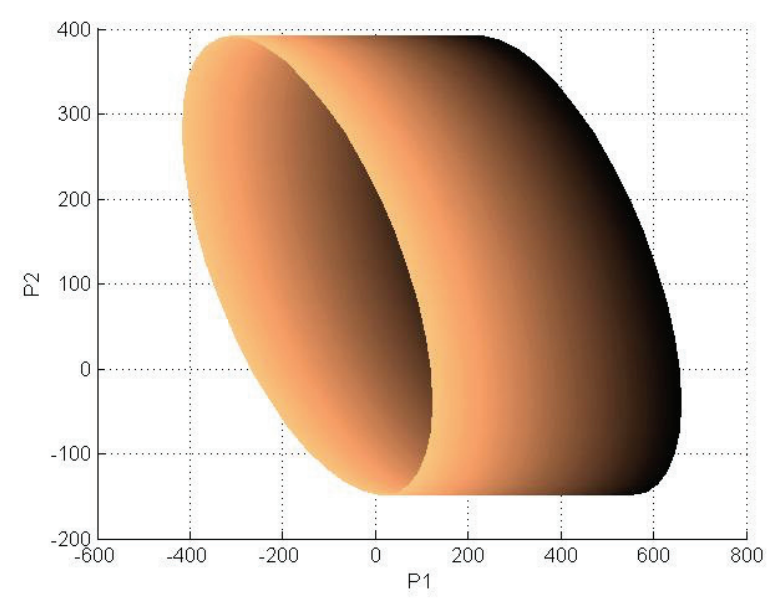

(b)

Fig. 6. Power surface of a 3-bus radial system (a) and power flow feasibility region obtained by using slack bus 3 (b)

otherwise, to the right. Hence, (8) can be considered as an infinite set of ellipses on the coordinate plane $P_{1} \times P_{2}$. Therefore, the power flow feasibility region of this power system is the area filled with ellipses (8). Its boundaries, i.e., marginal states, represent the envelopes of the infinite set of ellipses (8).

The maximum and minimum values of active power, which bus 1 can receive from slack bus 3 , are determined by the expression $P_{13}^{\max }=V_{1} V_{3} / X_{13}=\square P_{13}^{\min }$. Therefore, the external boundary of the power flow feasibility region is the boundary of the convex hull of two outermost ellipses displaced horizontally to the left and to the right by the power value $P_{13}^{\max }=V_{1} V_{3} / X_{13}$ with respect to the central ellipse (7), i.e., the closed curve $A-B-C-D-A$ in Fig. 4. The «hole» through the power flow feasibility region of this power system is the area of intersection of these two outermost ellipses, i.e., the region bounded by two arcs $E F$ of these outermost ellipses. It is interesting to notice that the power flow feasibility region of this power system will not have a «hole» if any other bus is assigned as a slack bus. For example, Fig. 5 demonstrates a power surface of the 3-bus radial power system with parameters $V_{1}=V_{2}=V_{3}=110 \mathrm{kV}, Z_{12}=20+j 40, Z_{13}=j 70(\Omega)$ and its power flow feasibility regions for different assigned slack buses.

According to Fig. 5(a), the power surface of the power system represents a hollow ellipsoidal cylinder. The projection of the power surface along the active power axis of bus 3 onto the coordinate plane $P_{1} \times P_{2}$, presented in Fig 5(b), has a form similar to that shown in Fig. 4 and represents a power flow feasibility region when bus 3 is used as a slack bus. If bus 1 is assigned as a slack bus, it corresponds to splitting the power system into two subsystems, whose steady states do not depend on each other. Steady states of the first subsystem are determined only by parameters of bus 2 , of the second subsystem by parameters of bus 3. It follows that the power flow feasibility region will be a filled rectangle, with the length of its sides determined by the maximum active power which bus 2 or bus 3 , respectively, can withdraw from the network and inject into it, as shown in Fig. 5(c). If bus 2 is assigned as a slack bus, the power flow feasibility region will be a filled parallelogram, Fig. 5(d).

If to reduce the inductive impedance $Z_{13}$, for example, to set $Z_{13}=j 45 \Omega$, i.e., to increase the electric connection of bus 1 with slack bus 3 , the power surface will also be represented as the hollow ellipsoidal cylinder (Fig. 6(a)), but projections of two outermost ellipses onto the coordinate plane $P_{1} \times P_{2}$ will not be crossed and the «hole» inside the power flow feasibility region will disappear, as shown in Fig. 6(b). The use of other slack buses does not qualitatively change power flow feasibility regions, and they will be similar to those presented in Figs. 5(c), (d).

\section{SLACK BUS}

The power surface allows a geometrical interpretation of the necessity of using the slack bus to calculate the steady state, i.e., the active power of the bus is not specified and regarded as unknown. Usually, such necessity is explained by the fact [30] that system losses are not known precisely before steady-state calculations. Therefore, it is impossible to specify power at all the buses precisely to provide consistency of the steady-state equations, i.e., their solvability. However, such consideration cannot explain the impossibility of calculating the steady state of a lossless power system with all $P V$-buses without using the slack bus. In a lossless power system, active power losses are equal to zero, therefore, it is not difficult to specify active power at all buses to provide consistency of the steadystate equations. However, even in this case, the steadystate calculation is impossible without assigning a slack bus. The power surface allows explaining such a need.

Each power system steady state corresponds to a certain point on the power surface and vice versa. The power surface is a map of the whole set of power system steady states, including all hypothetical (unstable) power system 
steady states, into the power space of buses. Outside and inside the power surface, there are no steady states. Like any surface, the power surface has a measure zero in the space of power at buses [31]. In terms of the probability theory, it means that a chance to specify the coordinates of a point on the power surface (the power of buses), disregarding their functional dependence so that they could satisfy the equation of the surface (steady state), is equal to zero. Hence, to obtain the coordinates of a point on the power surface (to solve the steady-state equations), it is necessary to use a coordinate of this point (active power of the slack bus) as a dependent variable. Therefore, all computational models that use steady-state equations always apply the slack bus explicitly or implicitly to calculate not only the steady state but also the optimal power flow, to estimate the power system state, and assess steady-state stability, and others [32]-[36].

\section{CONCLUSION}

The study of steady-state equations reveals that a set of the power system steady states can be geometrically represented as a surface in the space of active power of buses and reactive power of $P Q$ buses referred to as the power surface of the power system.

The findings indicate that the power flow feasibility region in the power space of buses, which is widely used in the theory and practice of power systems, is nothing but the projection of the power surface along the axis of the slack bus active power onto the subspace of all other specified power of buses, and the boundary of this projection is the surface of marginal states of the power system in the space of specified power of buses. Since the power surface is not plane, its projection, i.e., the power flow feasibility region, and its boundaries, i.e., the surface of marginal states, will depend on the choice of the axis along which it is projected, i.e., on the slack bus choice. The lossless power system power surface is plane, consequently, its power flow feasibility region and the surface of marginal states do not depend on the choice of slack bus.

The power surface allows visual interpretation of how «holes» through the power flow feasibility region appear and disappear in the case where the slack bus is changed.

Consideration of the set of all possible steady states of the power system as the power surface allows making use of the differential geometry and singularity theory for the further research of specificity and structure of power flow feasibility regions and marginal states.

\section{REFERENCES}

[1] F. Milano, Power System Modeling and Scripting, London: Springer-Verlag, 2010, 556 p.

[2] B.I. Ayuev, V.V. Davydov, P.M. Erokhin, V.G. Neuiymin, Steady State Computing Models in Power Systems, Moscow, Russia: Nauka, 2008, P. 256, (in Russian).

[3] A. Araposthatis, S. Sastry, P. Varaiya «Bifurcation analysis of the load flow equations,» in Proc. $19^{\text {th }}$
IEEE Conf. on Decision and Control, vol.1, pp. 641644, New Mexico, Dec. 1980.

[4] C.J. Tavora, O.J.M. Smith, «Equilibrium analysis of power systems,» IEEE Trans. PAS-91. vol. 91, no. 3, pp. 1131-1137, 1972.

[5] A.J. Korsak, «On the question of uniqueness of stable load-flow solutions,» IEEE Trans. PAS, vol. PAS-91, May/June 1972, pp. 1093-1100.

[6] A. Araposthatis, S. Sastry, P. Varaiya «Analysis of power-flow equation,» Electrical Power and Energy Systems, vol.3, no.3, pp. 115-126, July 1981.

[7] M.P. Rudnitsky, Theory Elements of Stability and Control of Steady States of Power Systems (in Russian). Sverdlovsk, USSR: UPI, 1984, 96 p.

[8] V.I. Idelchik, «Limit on the existence of a steady-state solution,» in Questions of Application of Methods at Control and Development of Power Systems» (in Russian), Irkutsk, USSR: Irkutsk Polytech. Inst. Press, 1975, pp.6-21.

[9] V.I. Tarasov, Theoretical Foundations for Analysis of the Load-Flow Problems in Electric Power Systems (in Russian). Novosibirsk: Nauka, 2002, 344 p.

[10] A.A. Vinogradov, V.I. Idelchik, A.I. Lazebnik, «Computational study of power flow feasibility region and the relationship between the limits of existence of a steady-state solution and static aperiodic stability,» in Questions of Application of Methods at Control and Development of Power Systems» (in Russian), Irkutsk, USSR, Irkutsk Polytech. Inst. Press, 1975, pp.22-49.

[11] V.P. Vasin, Power Flow Feasibility Regions of Power Systems (in Russian). Moscow, URSS: MEI, 1982, $84 \mathrm{p}$.

[12] Y.V. Makarov, Z.Y. Dong, D.J. Hill, «On convex of power flow feasibility boundary,» IEEE Trans. Power Syst. vol. 23, no. 2, May 2008, pp. 811-813.

[13] G.B. Price, «A generalized circle diagram approach for global analysis of transmission system performance,» IEEE Trans. PAS-103, vol. 103, no. 10, pp. 2881-2890, 1984.

[14] I.A. Hiskens, R.J. Davy, «Exploring the power flow solution space boundary,» IEEE Trans. Power Syst., vol. 16, no. 3, pp. 389-395, Aug. 2001.

[15] B.C. Lesieutre, I.A. Hiskens «Convexity of the set of feasible injections and revenue adequacy in FTR markets,» IEEE Trans. Power Syst., vol. 20, no. 4, pp. 1790-1798, Nov. 2005.

[16] B. Zhang, D. Tse, «Geometry of injection regions of power networks,» IEEE Trans. Power Syst., vol.28, no.2, pp. 788-797, 2013.

[17] J. Lavaei, D. Tse, B. Zhang, «Geometry of power flows and optimization in distribution networks,» IEEE Trans. Power Syst., vol.29, no.2, pp. 572-583, 2014.

[18] J. Jarjis, F.D. Galina, «Quantitative analysis of steady state stability in power systems,» IEEE Trans. PAS100. vol. 100, no. 1, pp. 318-326, 1981. 
[19] R. Seydel, From Equilibrium to Chaos: Practical Bifurcation and Stability Analysis. N.-Y.: Elsevier science publishing Co., 1988, 367 p.

[20] J.W. Bruce, P.G. Giblin, Curves and Singularities. A Geometrical Introduction to Singularity Theory. O.V. Cambridge: Cambridge University Press, 1984.

[21] V.I. Arnold, Catastrophe Theory, (in Russian), Moscow; USSR, Nauka, 1990, 128 p.

[22] T. Poston, I.N. Stewart, Catastrophe Theory and its Applications. Surveys and Reference Works in Math. 2, Pitman, London: 1978.

[23] M. Ortega, W.C. Rheinboldt, Iterative Solution of Nonlinear Equations in Several Variables. N.-Y. and London: Academic Press, 1970.

[24] O.V. Sterbachev, A.N. Zeiliger, K.P. Kadomskaya, a.o., Applying Digital Computers in Energy Industry (in Russian). Leningrad, USSR: Energiya, 1980, 237 p.

[25] A.M. Kontorovish, A.V. Kryukov, Power Systems Marginal States (Bases of Theory and Calculation Methods) (in Russian). Irkutsk, USSR: Irkutsk Polytech. Inst. Press, 1985, 72 p.

[26] B. I. Ayuev, V. V. Davydov, P. M. Erokhin, «Nonlinear-programming-based model of power system marginal states: Theoretical substantiation,» [Online]. Available: http//arxiv.org/abs/1505.03991, 2015.

[27] T.J. Overbye, «A power flow measure for unsolvable cases,» IEEE Trans. Power Syst., vol. 9, no. 3, pp. 1359-1365, Aug. 1994.

[28] T.J. Overbye, «Computation of a practical method to restore power flow solvability,» IEEE Trans. Power Syst., vol. 10, no. 1, pp. 280-287, Feb. 1995.

[29] V.V. Davydov, P.M. Erokhin, K.U. Kirilov, «Powers hypersurface of power system steady states,» (in Russian), in Proc. 3rd Int. Sci.\&Tech. Conf. Power Industry by Youth Eyes, Yekaterinburg, Russia, 2012, vol. 1, pp. 191-197.

[30] B. Stott, «Review of load-flow calculation methods,» Proc. IEEE, vol.62, no.7, pp. 916-929, July 1974.

[31] J.A. Thorpe, Elementary Topics in Differential Geometry. N.-Y.: Springer-Verlag Inc, 1979, 253 p.

[32] B.I. Ayuev, V.V. Davydov, P.M. Erokhin, «Fast and reliable method of searching power system marginal states,» IEEE Trans. Power Syst., vol. 31, no.6, pp. 4525-4533, Nov. 2016.

[33] B.I. Ayuev, V.V. Davydov, P.M. Erokhin, «Model of closest marginal states of power systems in p-norms,» IEEE Trans. Power Syst., vol. 33, no.2, pp. 1195-1208, Mar. 2018.

[34] V.V. Davydov, B.I. Ayuev, P.M. Erokhin, M.A. Prudov, «Studying a power system position model,» (in Russian), Elektrichestvo, no.3, pp. 4-14, 2019.

[35] A. Pazderin, S. Yuferev, «Power flow calculation by a combination of Newton-Raphson method and Newton's method in optimization,» in IECON Proc. 2009, 5414826, pp.1693-1696
[36] A. Pazderin, S. Yuferev, «Combined Newton's method for power flow calculation,» in Proc. $6^{\text {th }}$ IASTED Asian Conf. on Power and Energy Syst. AsiaPES 2013, pp. 104-107

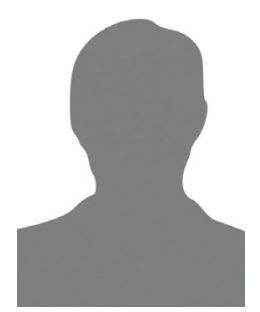

Boris I. Ayuev received the Dipl. Eng. and the Ph.D. degree in electrical engineering from Ural Federal University, Yekaterinburg, Russia, in 1979 and 1999 respectively, and Dr.Sc. degree in electrical engineering from Novosibirsk State Technical University, Novosibirsk, Russia in 2008.

$\mathrm{He}$ is now the Chairman of the JSC System Operator of the United Power System, Moscow, Russia. His research interests include control, operations, planning, and economics of power systems.

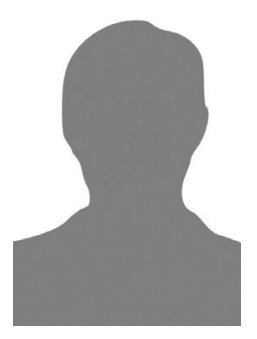

Viktor V. Davydov received the Dipl. Eng. in electrical engineering from East Siberian Technological Institute, Ulan-Ude, Russia, in 1979, the Ph.D. degree in electrical engineering from Ural Federal University, Yekaterinburg, Russia, in 1987, and the Dr.Sc. degree in electrical engineering from Novosibirsk State Technical University, Novosibirsk, Russia in 2019.

He is now a leading specialist of the JSC System Operator of the United Power System branch - ODU of Siberia, UlanUde, Russia. His research interests are steady state computing models, power system dynamics, control and stability, marginal states, and emergency control.

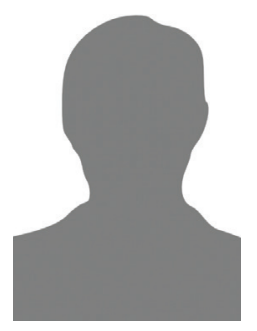

Petr M. Erokhin received the Dipl. Eng., the Ph.D. and Dr.Sc. degrees in electrical engineering from Ural Federal University, Yekaterinburg, Russia in 1969, 1975, and 2005 respectively.

$\mathrm{He}$ is now a Professor of Electrical Engineering at Ural Federal University, Yekaterinburg, Russia. His research interests include control, operations, planning, and economics of power systems. 


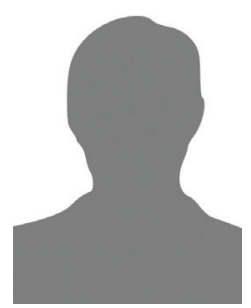

Vladimir G. Neuymin received the Dipl.Eng. and the Ph.D. degree in electrical engineering from Ural Federal University, Yekaterinburg, Russia in 1982, 1987, respectively.

He is now a Head of Department of JSC "Scientific and Technical Center of Unified Power System," Yekaterinburg, Russia. His research interests include control and stability, steady states computing models, power system dynamics, state estimation, planning, and economics of power systems.

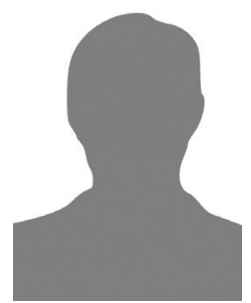

Andrey V. Pazderin received the Dipl. Eng., the Ph.D. and Dr.Sc. degrees in electrical engineering from Ural Federal University, Yekaterinburg, Russia in 1982, 1987, and 2005 respectively.

$\mathrm{He}$ is now a Professor of Electrical Engineering at Ural Federal University, Yekaterinburg, Russia. His research interests include control and stability, operations, state estimation, planning, and economics of power systems. 